\title{
Introduction of quantitative and qualitative cornea optical coherence tomography findings induced by collagen cross-linking for keratoconus: a novel effect measurement benchmark
}

\author{
This article was published in the following Dove Press journal: \\ Clinical Ophthalmology \\ 13 February 2013 \\ Number of times this article has been viewed
}

\section{A John Kanellopoulos 1,2 George Asimellis' \\ 'Laservision.gr Institute, Athens, Greece; ${ }^{2} \mathrm{New}$ York University Medical School, New York, NY, USA}

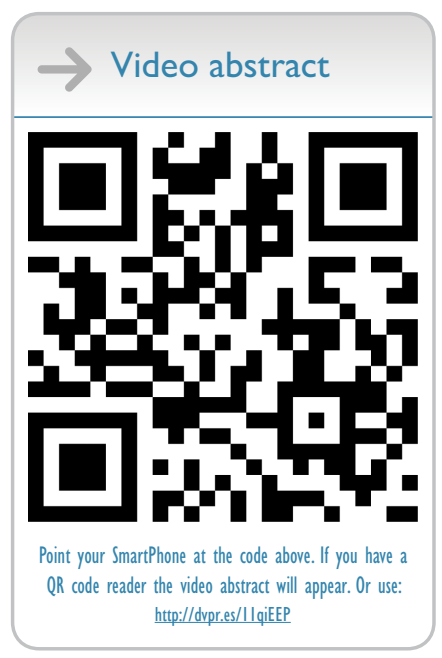

Correspondence: A John Kanellopoulos Laservision.gr Eye Institute, 17 Tsocha Street, Athens II52I, Greece

Tel +302107472777

Fax +30210747 2789

Email ajk@brilliantvision.com
Purpose: To introduce a novel, noninvasive technique to determine the depth and extent of anterior corneal stroma changes induced by collagen cross-linking (CXL) using quantitative analysis of high-resolution anterior-segment optical coherence tomography (OCT) post-operative images.

Setting: Private clinical ophthalmology practice.

Patients and methods: Two groups of corneal cross-sectional images obtained with the OptoVue RTVue anterior-segment OCT system were studied: group A (control) consisted of unoperated, healthy corneas, with the exception of possible refractive errors. The second group consisted of keratoconic corneas with CXL that were previously operated on. The two groups were investigated for possible quantitative evidence of changes induced by the CXL, and specifically, the depth, horizontal extent, as well as the cross-sectional area of intrastromal hyper-reflective areas (defined in our study as the area consisting of pixels with luminosity greater than the mean $+2 \times$ standard deviation of the entire stromal cross section) within the corneal stroma.

Results: In all images of the second group (keratoconus patients treated with CXL) there was evidence of intrastromal hyper-reflective areas. The hyper-reflective areas ranged from $0.2 \%$ to $8.8 \%$ of the cross-sectional area (mean \pm standard deviation; $3.46 \% \pm 1.92 \%$ ). The extent of the horizontal hyper-reflective area ranged from $4.42 \%$ to $99.2 \%(56.2 \% \pm 23.35 \%)$ of the cornea image, while the axial extent (the vertical extent in the image) ranged from $40.00 \%$ to $86.67 \%(70.98 \% \pm 7.85 \%)$. There was significant statistical difference $(P<0.02)$ in these values compared to the control group, in which, by application of the same criteria, the same hyper-reflective area (owing to signal noise) ranged from $0.00 \%$ to $2.51 \%(0.74 \% \pm 0.63 \%)$.

Conclusion: Herein, we introduce a novel, noninvasive, quantitative technique utilizing anterior segment OCT images to quantitatively assess the depth and cross-sectional area of CXL in the corneal stroma based on digital image analysis. Mean cross-sectional area showing evidence of CXL was $3.46 \% \pm 1.92 \%$ of a $6 \mathrm{~mm}$ long segment.

Keywords: Collagen cross-linking, keratoconus, optical coherence tomography, higher fluence cross-linking, cornea ectasia, Athens Protocol

\section{Introduction}

Keratoconus $(\mathrm{KCN})$ is a degenerative bilateral, progressive, noninflammatory disorder characterized by ectasia, thinning, and increased curvature of the cornea, and is associated with loss of visual acuity, particularly in relation to high-order aberrations. $^{1-4}$ 
Corneal collagen cross-linking (CXL) with riboflavin and ultraviolet-A irradiation is a common technique for tissue stabilization. 5,6 Several studies have shown that CXL is an effective intervention to halt the progression of keratoconus and corneal ectasia. ${ }^{7}$

Anterior-segment optical coherence tomography (AS-OCT) is a promising imaging mode providing highresolution cross-sectional images across a meridian of choices that can be employed in $\mathrm{KCN}$ diagnosis. ${ }^{8,9}$ The most advanced AS-OCT systems invariably employ Fourier spectral-domain signal processing. As of today, there are a number of different spectral domain OCT systems commercially available. ${ }^{10,11}$

The ability to provide real-time cross sectional mapping, in conjunction with the very principle of operation, namely photon back scattering, provides the understudied application of quantitative assessment of the extent of stromal changes due to CXL.

\section{OCT and CXL demarcation line observations}

To date, the efficacy of CXL treatment can be monitored only indirectly by postoperative follow-up observations, such as with a Scheimpflug camera, ${ }^{12}$ or with corneal confocal microscopy. ${ }^{13}$

In addition, a corneal stromal demarcation line indicating the transition zone between cross-linked anterior corneal stroma and untreated posterior corneal stroma can be detected in slit-lamp examination as early as 2 weeks after treatment. ${ }^{14}$ In our clinical assessment, the presence of this finding over the anterior two-thirds of the stroma confirms that sufficient CXL treatment has occurred.

Following our presentation and the introduction in the peer-reviewed literature of the use of OCT imaging in order to evaluate the CXL-induced demarcation line, OCT has seen some recent interest as a tool for investigating CXL effects, such as corneal thickness before and after CXL for $\mathrm{KCN}$, and demarcation line depth following CXL. ${ }^{15-22}$

The principle lies in the fact that although these lines do not appear to affect vision, as they correspond to changes in stromal density, they appear as brighter (hyper-reflective) areas on cross-sectional corneal OCT scans. However, the depth and extent of stromal changes induced by CXL has been difficult to evaluate quantitatively in the clinic.

The motivation for our study was to advance this aforementioned theory by examining not only the demarcation line depth between the suspected CXL and the deeper cornea with corneal OCT, but also to attempt to quantitatively assess the extent of this area on a large number of patients over a large postoperation interval. Our novel technique is based on digital signal processing on cross-sectional OCT images of corneas, and evaluates quantitatively and, in our opinion, free of examiner bias, the extent of CXL changes in the corneal stroma.

\section{Methods}

This prospective interventional case series study received approval by the Ethics Committee of our Institution and adhered to the tenets of the Declaration of Helsinki. Informed consent was obtained from each subject at the time of the CXL intervention or at the first clinical visit. The study was conducted in our clinical practice on patients during their regular clinical visits (control group) and scheduled postoperative procedure visits (KCN group).

\section{Patient inclusion criteria}

The control group (50 patients, 100 eyes) consisted of patients with eyes with unoperated corneas (ie, normal eyes with no ocular pathology other than refractive error). Mean patient age was $35.2 \pm 9.1$ years (range 19-48), equally divided between males and females. Before OCT corneal mapping, a complete ocular examination and tomographic topography was performed to screen for corneal abnormalities.

The second group (47 patients, 94 eyes) consisted of $\mathrm{KCN}$ patients previously operated with CXL by employing the Athens Protocol, which combined same-day phototherapeutic keratectomy epithelial removal and partial topographicallyguided photorefractive keratectomy normalization of the cornea ectasia, followed by high-fluence, short-duration riboflavin induced CXL. ${ }^{21}$

The mean patient age in this group was $28.1 \pm 7.1$ years (range 16-45 years). There is a bias towards males in this group (33 males, 14 females), which is consistent with our clinical experience of the male-female incidence of keratoconic
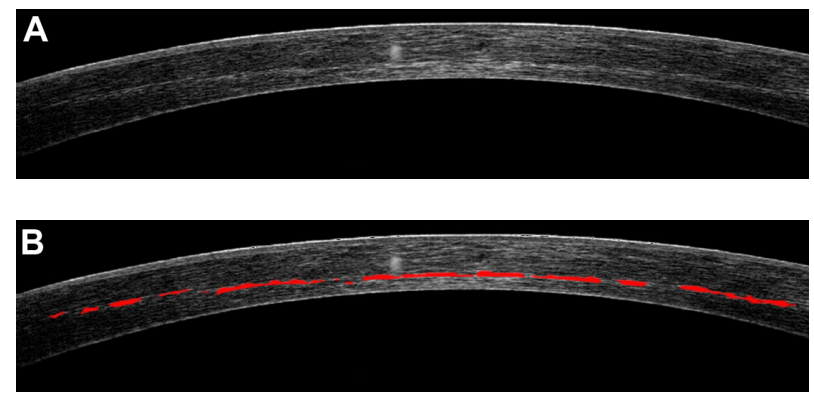

Figure I (A) Typical cornea cross-sectional meridian image of a patient with KCN. (B) The selected hyper-reflective intrastromal area is indicated in red. 
Table I Hyper-reflective area corresponding to meridional corneal cross section, as measured in the two groups

\begin{tabular}{|c|c|c|c|c|}
\hline & \multicolumn{2}{|c|}{ Group A (control) } & \multicolumn{2}{|c|}{ Group B (KCN) } \\
\hline & $\begin{array}{l}\text { Hyper- } \\
\text { reflective } \\
\text { area (pixels) }\end{array}$ & $\begin{array}{l}\text { Cross- } \\
\text { sectional } \\
\text { area (\%) }\end{array}$ & $\begin{array}{l}\text { Hyper- } \\
\text { reflective } \\
\text { area (pixels) }\end{array}$ & $\begin{array}{l}\text { Cross- } \\
\text { sectional } \\
\text { area (\%) }\end{array}$ \\
\hline Mean & 427 & $0.74 \%$ & 2018 & $3.46 \%$ \\
\hline Max & 1518 & $2.50 \%$ & 4927 & $8.80 \%$ \\
\hline Min & 0 & $0.00 \%$ & 121 & $0.18 \%$ \\
\hline Stdev & 374 & $0.63 \%$ & 1105 & $1.92 \%$ \\
\hline
\end{tabular}

patients, and with previous reports. ${ }^{2}$ Of the 94 eyes included in the study, 47 were oculus sinister and 47 were oculus dexter. Mean postoperative time since CXL operation was $17.65 \pm 20.83$ months, with a range of 1 to 72 months.

Most patients enrolled in the group had bilateral CXL operation, and thus both eyes were included in the study, while in some patients only one eye was included in the study. For some patients, images from more than one visit were included in the study (separated by at least 3 months).

\section{Materials}

The OptoVue RTVue (OptoVue Inc, Fremont, CA, USA) AS-OCT system was employed in the study. Using the L-Cam lens, a $6 \mathrm{~mm}$ long Hi-Res Cross Line Scan, centered at the pupil center along the vertical meridian, was recorded. The meridional cross-sectional images were processed with the RTVue software (version 5.1.0, processing algorithm A5, 1, 0, 90). The software averages up to 32 successive acquisitions. In our study, we included images consisting of at least five averages.

\section{Our novel investigation technique}

All images from both groups were investigated for possible quantitative evidence of changes induced by CXL. Evidence of such was considered as the existence of the intrastromal hyper-reflective demarcation line. To search for such a line, images were loaded into commercially available software, Adobe Photoshop CS5 Version 12.04 (Adobe Systems Inc, San Jose, CA, USA).

For every meridional cross-sectional image, the pixels associated with the stromal cross-section were selected with the marquee tool. The area separated by 10 pixels from the anterior and the exterior corneal surfaces were deselected, as they are typically of higher luminosity (Figure 1). The extent (pixel count) of the selected stromal image area was determined with the histogram tool report. The dialog box for this tool also provides the mean \pm standard deviation of the luminosity for the selected area.

The hyper-reflective demarcation area was quantitatively defined in this study as the population of pixels (pixel count) having luminosity greater than the value defines as luminosity mean $+2 \times$ standard deviations, as obtained in the previous step.

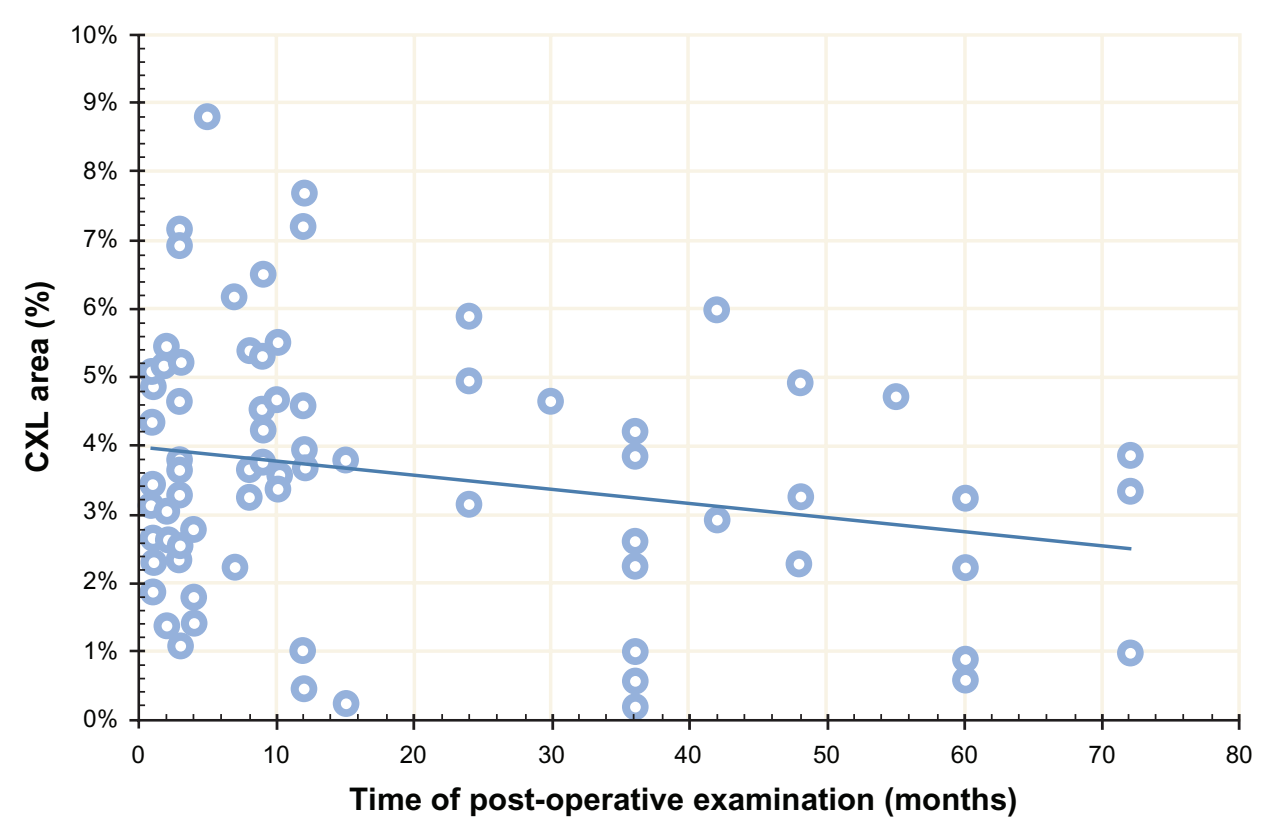

Figure 2 Demarcation line area as a function of time elapsed since the CXL operation.

Note: The trend line shows that over time, this area diminishes.

Abbreviation: CXL, cross-linking. 
Table 2 Horizontal and axial extent of hyper-reflective area in group $\mathrm{B}(\mathrm{KCN})$

\begin{tabular}{|c|c|c|c|c|c|}
\hline & \multirow[t]{2}{*}{$\begin{array}{l}\text { CXL width } \\
\text { (pixels) }\end{array}$} & \multirow{2}{*}{$\begin{array}{l}\text { Horizontal } \\
\text { extent } \\
\text { Overall width }\end{array}$} & \multirow[t]{2}{*}{$\begin{array}{l}V \text { line } \\
\text { total }\end{array}$} & \multirow{2}{*}{$\begin{array}{l}\text { Axial extent } \\
\text { V line CXL }\end{array}$} & \multirow[t]{2}{*}{$\% \mathbf{V}$} \\
\hline & & & & & \\
\hline Mean & 483.35 & $56.20 \%$ & 61.93 & 43.81 & $70.98 \%$ \\
\hline Max & 853 & $99.19 \%$ & 80 & 65 & $86.67 \%$ \\
\hline Min & 38 & $4.42 \%$ & 38 & 30 & $40.00 \%$ \\
\hline Stder & 200.82 & $23.35 \%$ & 8.18 & 6.96 & $7.85 \%$ \\
\hline
\end{tabular}

Abbreviations: $\mathrm{KCN}$, keratoconus; $\mathrm{CXL}$, cross-linking; $\mathrm{V}$, vertical; Max, maximum; Min, minimum; Stdev, standard deviation.

Subsequently, after using the histogram tool report again, the extent (pixel count) of the hyper-reflective intrastromal area was recorded, as well as its horizontal extent (pixels across the y axis), and this was compared to the horizontal extent of the captured image, which was set to a standard of 860 pixels across the $y$-axis.

Similarly, the axial extent (depth of demarcation line) was assessed and compared to the depth of the corneal section (vertical line in the image; that is, pixels in the $\mathrm{x}$-axis) to which it corresponded.

Descriptive statistics (average, minimum, maximum, standard deviation, bias, and range), comparative statistics, and linear regression were performed in Microsoft Excel 2010 (Microsoft Corp, Redmond, WA, USA) and OriginLab version 8 (OriginLab Corp, Northampton, MA, USA). Analysis of variance between groups was performed using the OriginLab statistics tool.

\section{Results}

\section{Areal extent (depth and diameter) of demarcation}

Cross-sectional meridian area measurements had an average of 59,183 pixels ( \pm 5778 ), ranging from 80,729 to 39,951 (maximum to minimum). This corresponds to an area of $2.88 \mathrm{~mm}^{2}$. Mean luminosity values were, on a grayscale of 0 to $255,63 \pm 13$, ranging from 89 to 25 (maximum to minimum).

As shown in Table 1, the intrastromal hyper-reflective area found with this technique for group A (control) had a mean area of $427.25 \pm 373.81$ pixels (range, maximum to minimum, $1518-0$ ), corresponding to a mean of $0.74 \% \pm 0.63 \%$ (range, $2.50 \%-0.00 \%$ ), corresponding to $0.02 \mathrm{~mm}^{2}$. This contrasts with group $\mathrm{B}(\mathrm{KCN})$, in which the mean hyper-reflective area had a mean of 2018.21 \pm 1104.70 (range, 4927-121), corresponding to a mean of $3.46 \% \pm 1.92 \%$ (range, $8.80 \%-0.18 \%$ ), or $0.098 \mathrm{~mm}^{2}$ of the corneal cross-sectional area. Of the 94 cases examined, 72 had more than $2.50 \%$ hyper-reflective areas, whereas six were close to and 16 were below this mark. The two groups were found to be statistically different (comparison of hyper-reflective area pixel count; $P<0.02$ ). The over time development of the extent of the area of demarcation; that is, the CXL area over post-operative time, is plotted in Figure 2.

\section{Horizontal extent of demarcation}

The horizontal extent of demarcation was assessed for the $\mathrm{KCN}$ group and was compared to the standard 860 pixel

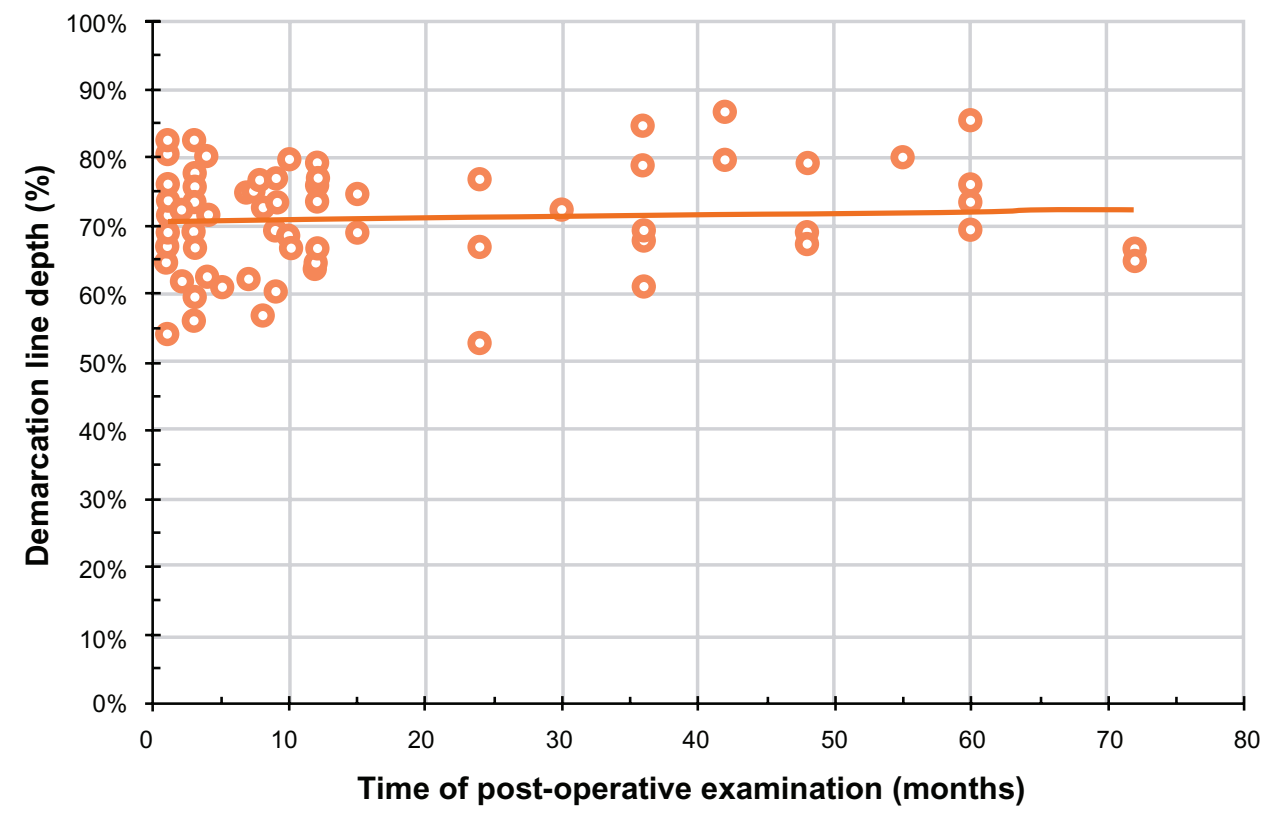

Figure 3 Demarcation line depth as a function of time elapsed since the CXL operation.

Note: The trend line shows that over time this depth remains constant to about two-thirds of the corneal depth.

Abbreviation: CXL, cross-linking. 


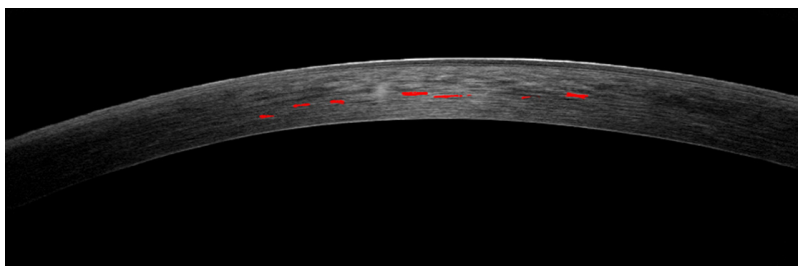

Figure 4 Example of epithelium-on CXL cornea with minimal appearance of the demarcation line.

Note: Of the 61,770 pixels (cornea cross-section), only 443 correspond to a hyperreflective area.

Abbreviation: CXL, cross-linking.

width extent of the corneal cross-section. As shown in Table 2, on average the extent of the hyper-reflective area (CXL width) was $483.35 \pm 200.82$ pixels (range, 853-38), corresponding to an average of $56.20 \%$ of the cross-section width, ranging from $99.19 \%$ to $4.42 \%$. Of the subgroup of 78 corneas with more than a $2.50 \%$ hyper-reflective area, the minimum was $12.45 \%$.

\section{Axial extent (depth) of demarcation}

The axial extent of demarcation corresponds to what we describe as the depth of the CXL effect. The quantitative assessment is subject to the corneal thickness, which varies significantly among images. In each image studied, the corneal thickness was measured in pixels (vertical line total in Table 2), and was found to correspond to an average of $61.93 \pm 8.18$ pixels ( $\max$ to $\min , 80-38$ ). Considering that $6 \mathrm{~mm}$ across the image corresponded to 860 pixels, the 61.93 pixels corneal thickness translates to $432 \mu \mathrm{m}$ of thickness.

Having measured the corneal thickness of each individual section, the distance in pixels (vertical line CXL) from the anterior corneal surface was measured. On average, it was found to be $43.81 \pm 6.96$ pixels (range, $65-30$ ), corresponding to $305.6 \mu \mathrm{m}$ or $70.98 \%$ of the total corneal thickness. The over time (postoperative) development of the depth of the area of demarcation, that is CXL area over time, is presented in Figure 3.

\section{Discussion}

By examining high-resolution corneal OCT images, we encountered statistically different findings between the treated group (KCN; group $\mathrm{B}$ ) and the control group (A).

It appears that there is a statistically significant difference between the control group and the KCN group regarding the presence of a demarcation line, as quantitatively measured by the extent of the area of the hyper-reflective demarcation line, indicating a localized change in stromal (treated) density over the underlying (untreated) stroma.

In 72 of 94 cases, the demarcation line area corresponded to more than $2.50 \%$ of the total corneal cross-sectional area, with a mean \pm standard deviation of $3.46 \% \pm 1.92 \%$. In the entire control group A, by applying the same luminosity criteria, the similar area had a mean of $0.74 \% \pm 0.63 \%$. We believe that these pixel counts represent merely signal noise rather than reflect actual changes in stromal density. Thus, we can ascertain that the demarcation line viewed by OCT can be a good indication of the extent of collagen density changes induced by CXL.

Over time, these density changes become less apparent. The trend line shown in Figure 2 has a negative slope (reduced

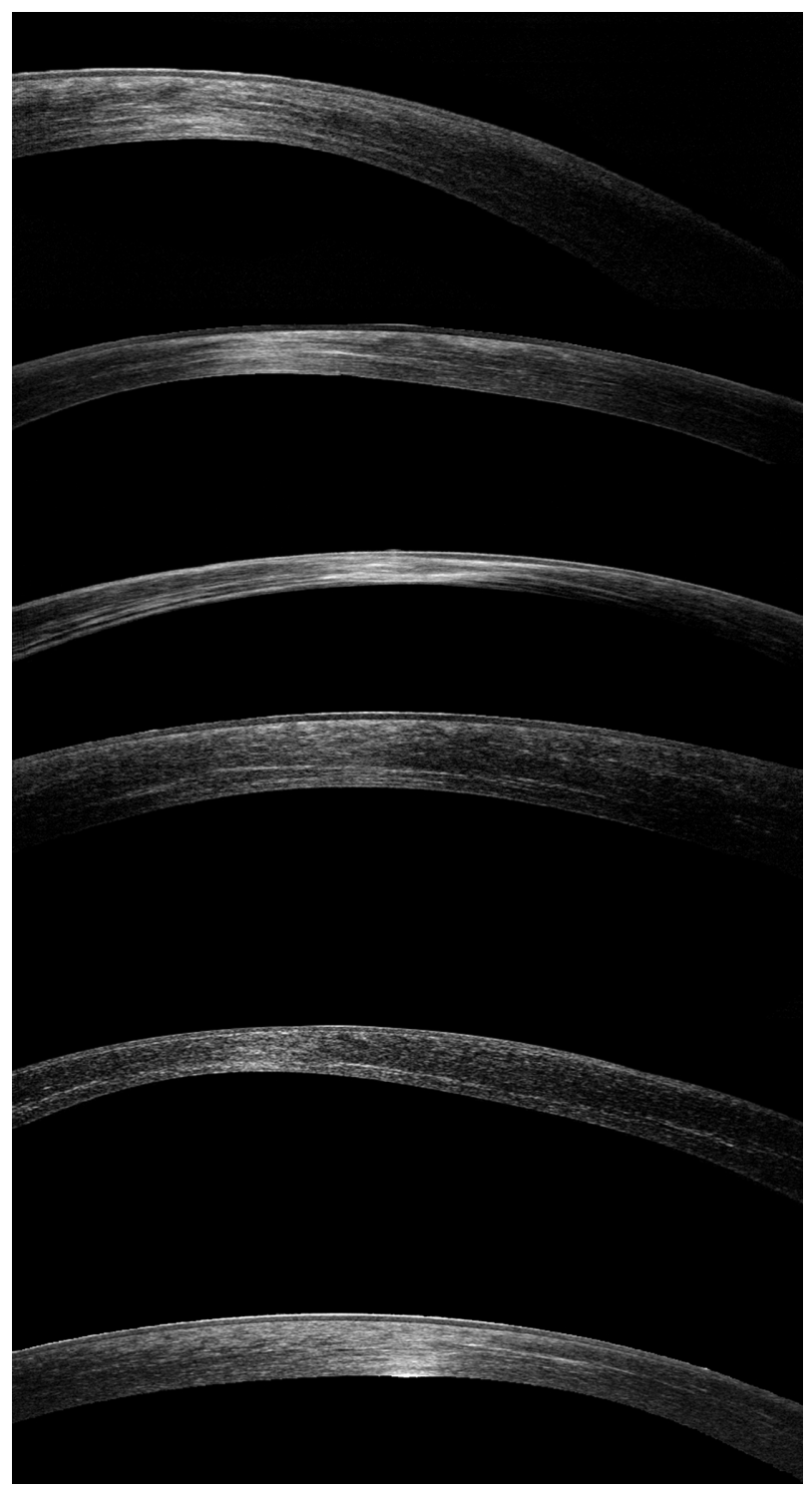

Figure 5 Example of corneal cross-sectional images examined in the study showing various degrees of demarcation line extent. 
by $0.02 \%$ per month), indicating that the demarcation line area fades away by postoperative month 12 , in agreement with our clinical findings. ${ }^{15}$

The depth of the demarcation line, found to be on average of $305 \mu \mathrm{m}$, is consistent with the accepted notion that in order to avoid ultraviolet-A irradiation damage to the corneal endothelium, ${ }^{23}$ the CXL parameters are set in a way that effective treatment occurs only in the first $300 \mu \mathrm{m}$ of the corneal stroma. ${ }^{12}$

The depth of the demarcation line appears, on the other hand, to be stable over time, even after 3 years following operation, at approximately $70 \%$ of the corneal depth. However, a deeper demarcation line depth (relative to the corneal depth) is associated with thinner corneal thickness, as measured postoperatively. In the selected 12 thinner corneas, the depth of the demarcation line was found to be $83 \%$ of the total corneal thickness.

One clinical example of ineffective CXL action is demonstrated in Figure 4, in which a case of a cornea treated in another institution with epithelium-on CXL technique demonstrated minimal signs of hyper-reflective areas. ${ }^{24}$ This case, which was not part of the case study, was presented to our practice with progressive ectasia following the CXL operation in another practice. Examples of corneal cross-sectional images examined in the study showing various degrees of demarcation line extent are presented in Figure 5.

\section{Conclusion}

AS-OCT appears to demonstrate reproducible early (1 month) and long-term (up to 3 years) CXL cornea findings. The hyper-reflective lines may represent induced cornea density changes or subtle intrastromal cornea scarring. This novel quantitative and qualitative technique may constitute a possible benchmark for a noninvasive measurement to evaluate and titrate the amount, extent, and depth of intrastromal effects of the CXL treatment in $\mathrm{KCN}$ and possibly ectasia eyes.

\section{Disclosure}

The authors report no conflicts of interest in this work.

\section{References}

1. Krachmer JH, Feder RS, Belin MW. Keratoconus and related noninflammatory corneal thinning disorders. Surv Ophthalmol. 1984;28(4):293-322.

2. Belin MW, Asota IM, Ambrosio R Jr, Khachikian SS. What's in a name: keratoconus, pellucid marginal degeneration, and related thinning disorders. Am J Ophthalmol. 2011;152(2):157-162.
3. Ambrósio R Jr, Caldas DL, da Silva RS, Pimentel LN, de Freitas VB. Impact of the wavefront analysis in refraction of keratoconus patients. Rev Bras Oftalmol. 2010;69(5):294-300. Portuguese.

4. Kosaki R, Maeda N, Bessho K, et al. Magnitude and orientation of Zernike terms in patients with keratoconus. Invest Ophthalmol Vis Sci. 2007;48(7):3062-3068.

5. Gkika M, Labiris G, Kozobolis V. Corneal collagen cross-linking using riboflavin and ultraviolet-A irradiation: a review of clinical and experimental studies. Int Ophthalmol. 2011;31(4):309-319.

6. Dahl BJ, Spotts E, Truong JQ. Corneal collagen cross-linking: an introduction and literature review. Optometry. 2012;83(1):33-42.

7. Jankov Ii MR, Jovanovic V, Delevic S, Coskunseven E. Corneal collagen cross-linking outcomes: review. Open Ophthalmol J. 2011;5:19-20.

8. Li Y, Meisler DM, Tang M, et al. Keratoconus diagnosis with optical coherence tomography pachymetry mapping. Ophthalmology. 2008;115(12):2159-2166.

9. Li Y, Tan O, Brass R, Weiss JL, Huang D. Corneal epithelial thickness mapping by fourier-domain optical coherence tomography in normal and keratoconic eyes. Ophthalmology. 2012;119(12):2425-2433.

10. Kiernan DF, Mieler WF, Hariprasad SM. Spectral-domain optical coherence tomography: a comparison of modern high-resolution retinal imaging systems. Am J Ophthalmol. 2010;149(1):18-31.

11. Grewal DS, Brar GS, Jain R, Grewal SP. Comparison of Scheimpflug imaging and spectral domain anterior segment optical coherence tomography for detection of narrow anterior chamber angles. Eye (Lond). 2011;25(5):603-611.

12. Kránitz K, Kovács I, Miháltz K, et al. Corneal changes in progressive keratoconus after cross-linking assessed by Scheimpflug camera. J Refract Surg. 2012;28(9):645-649.

13. Filippello M, Stagni E, Buccoliero D, Bonfiglio V, Avitabile T. Transepithelial cross-linking in keratoconus patients: confocal analysis. Optom Vis Sci. 2012;89(10):e1-e7.

14. Seiler T, Hafezi F. Corneal cross-linking-induced stromal demarcation line. Cornea. 2006;25(9):1057-1059.

15. Kanellopoulos AJ. Novel cornea OCT findings in early and long-term follow-up of collagen crosslinking for keratoconus. Proceedings of the American Academy of Ophthalmology; October 24-27, 2009; San Francisco, CA, USA.

16. Kanellopoulos AJ. Comparison of sequential vs same-day simultaneous collagen cross-linking and topography-guided PRK for treatment of keratoconus. J Refract Surg. 2009;25(9):S812-S818.

17. Kanellopoulos AJ. The management of cornea blindness from severe corneal scarring, with the Athens Protocol (transepithelial topographyguided PRK therapeutic remodeling, combined with same-day, collagen cross-linking). Clin Ophthalmol. 2012;6:87-90.

18. Mencucci R, Paladini I, Virgili G, Giacomelli G, Menchini U. Corneal thickness measurements using time-domain anterior segment OCT, ultrasound, and Scheimpflug tomographer pachymetry before and after corneal cross-linking for keratoconus. J Refract Surg. 2012;28(8):562-566.

19. Doors M, Tahzib NG, Eggink FA, Berendschot TT, Webers CA, Nuijts RM. Use of anterior segment optical coherence tomography to study corneal changes after collagen cross-linking. Am J Ophthalmol. 2009;148(6):844-851.

20. Yam JC, Chan CW, Cheng AC. Corneal collagen cross-linking demarcation line depth assessed by Visante OCT after CXL for keratoconus and corneal ectasia. J Refract Surg. 2012;28(7): 475-481.

21. Ortiz S, Pérez-Merino P, Alejandre N, Gambra E, Jimenez-Alfaro I, Marcos S. Quantitative OCT-based corneal topography in keratoconus with intracorneal ring segments. Biomed Opt Express. 2012;3(5): 814-824.

22. Kanellopoulos AJ. Long term results of a prospective randomized bilateral eye comparison trial of higher fluence, shorter duration ultraviolet A radiation, and riboflavin collagen cross linking for progressive keratoconus. Clin Ophthalmol. 2012;6:97-101. 
23. Bagga B, Pahuja S, Murthy S, Sangwan VS. Endothelial failure after collagen cross-linking with riboflavin and UV-A: case report with literature review. Cornea. 2012;31(10):1197-1200.
24. Stojanovic A, Chen X, Jin N, et al. Safety and efficacy of epithelium-on corneal collagen cross-linking using a multifactorial approach to achieve proper stromal riboflavin saturation. J Ophthalmol. 2012;2012:498435.
Clinical Ophthalmology

\section{Publish your work in this journal}

Clinical Ophthalmology is an international, peer-reviewed journal covering all subspecialties within ophthalmology. Key topics include: Optometry; Visual science; Pharmacology and drug therapy in eye diseases; Basic Sciences; Primary and Secondary eye care; Patien Safety and Quality of Care Improvements. This journal is indexed on

Submit your manuscript here: http://www.dovepress.com/clinical-ophthalmology-journal

\section{Dovepress}

PubMed Central and CAS, and is the official journal of The Society of Clinical Ophthalmology (SCO). The manuscript management system is completely online and includes a very quick and fair peer-review system, which is all easy to use. Visit http://www.dovepress.com/ testimonials.php to read real quotes from published authors. 